\title{
The pharmacodynamic inoculum effect from the perspective of bacterial population modeling
}

\author{
Desiree Y. Baeder ${ }^{1}$ and Roland R. Regoes ${ }^{1}$ \\ ${ }^{1}$ Institute of Integrative Biology, ETH Zurich, Universitätsstrasse 16, 8092 Zürich, \\ Switzerland
}

\section{Abstract}

2 The quantitative determination of the effect of antimicrobials is essential for

4 codynamics, and for predictive mathematical modeling. It is well-established

5 that the effect of antimicrobials depends on the bacterial inoculum size used

6 in assays - a phenomenon called inoculum effect. The commonly used phar-

7 macodynamic (PD) function that captures the antimicrobial effect, however,

8 is derived from assays conducted with one bacterial inoculum size only. Here,

9 we used the mathematical multi-hit model, which describes antimicrobial action mechanistically, to quantify the effect of the inoculum on the PD parameters. Based on our quantitative analysis, we propose an extended PD function that incorporates the inoculum effect. The extended PD function could mimic the long-term population dynamics for reversibly binding antimicrobials, but failed when antimicrobials were also assumed to be degraded. We 
further show that, in simulation with competing antimicrobial-sensitive and -resistant bacteria, neglecting the inoculum effect leads to an overestimation of the competitive ability of the resistant strain. Our work emphasizes that the PD function - and in general any efficacy measure, such as the MIC should include information about the inoculum size on which it is based, and ideally account for the inoculum effect.

Keywords: inoculum effect, pharmacodynamic function, multi-hit model, antimicrobial efficacy, MIC, bacterial population dynamics

\section{Introduction}

Efficacy of antimicrobials against bacteria is quantified using time-kill curves and is subsequently captured in so-called pharmacodynamic (PD) functions, also known as $E_{\max }$ and Zhi models $(1,2,3)$. A time-kill curve captures how a bacterial population changes over time in presence of a given concentration of an antimicrobial (Fig. 1a). During exponential growth, time-kill curves are approximately linear on a logarithmic scale. Thus, the slopes of these loglinear time-kill curves describe the change of the bacterial population over time and are a direct measure of the bacterial net growth rate. The PD function returns the net growth rate for any given antimicrobial concentration. The parameters of the PD function are estimated based on several time-kill curves differing in the antimicrobial concentration, but with the same initial 
bacterial population size (bacterial inoculum) $(1,4,5,6,7,8)$.

A more widespread but less informative way to express efficacy of antimicrobials is the minimum inhibitory concentration (MIC). The MIC is the lowest drug concentration that prevents visible bacterial growth (Fig. 1b). For concentration-independent antimicrobials (steep PD curve), the MIC is comparable to the antimicrobial concentration that results in a time-kill curve with the slope of zero (8). In this case, one of the four parameters of the $\mathrm{PD}$ function, which we call $M I C_{P D}$, is equivalent to the MIC. Although it is recognized that the MIC might change due to change in bacterial inoculum size (see for example the MIC protocol by Wiegand et al. (9)), both the CLSI and the EUCAST recommendation to measure the MIC is based on a standard bacterial inoculum of $5 \times 10^{5} \mathrm{CFU} / \mathrm{ml}$ only $(10,11)$. Thus, as in the case of time-kill curves, standardization of the inoculum neglects any effect on efficacy of antimicrobials due to changes in the bacterial inoculum.

Both experimental and theoretical work has put forward hypotheses explaining why the inoculum size matters when determining the efficacy for antimicrobials targeting exponentially growing bacteria. Maybe the most intuitive explanation is that the amount of antimicrobials is finite in the system and a decrease in the amount of free antimicrobials changes the efficacy of the antimicrobial $(12,13,14)$. The amount of antimicrobials decreases over time because of binding processes. Assuming mass action kinetics, the number of free antimicrobial molecules is reduced proportional to bacterial cell density, which results in changes in the efficacy of the antimicrobial. In addition to 
this binding kinetic effect, bacteria can produce extracellular enzymes that further decrease the concentration of antimicrobials. For example, $\beta$-lactam antibiotics are degraded by $\beta$-lactamases $(15,16,17,18,19,20,21,22)$, and antimicrobial peptides can be degraded by proteases $(23,24,25)$. The higher the bacterial population, the more enzymes are produced and thus the higher the change in antimicrobial efficacy.

Changes in efficacy of antibacterial drugs caused by changing the initial bacterial inoculum size are summarized under the term inoculum effect (Fig. 1). The inoculum effect was first described in the early 1940s (20). Since then, the inoculum effect has been measured experimentally for a variety of combinations of bacterial species and antibiotics $(13,17,18,21,22,26,27$, 28). Recently, the inoculum effect became of interest in the field of antimicrobial peptides (AMPs) $(29,30)$. It is common to determine the inoculum effect in terms of the change in the MIC with inoculum size $(13,17,22,26,27,29)$. To our knowledge, the inoculum effect has not yet been determined using the more comprehensive PD function approach. In addition to its experimental quantification in the wet lab, the inoculum effect has been explored with mathematical models. These models either comprise a mechanistic description of the interaction between antimicrobial molecules and bacterial cells $(12,14,28,31,32)$ or are based directly on MIC measurements (13). In the latter case, the inoculum effect was deterined by measuring the MIC at a variation of bacterial inoculum sizes. The measured effect was then incorporated by extending the PD function for the $M I C_{P D}$ parameter. 
Here we use mathematical modeling as a tool to explore how bacterial inoculum size affects all four parameters of the PD function. The advantage of employing mathematical models is the possibility to determine the efficacy of an antimicrobial for various bacterial inoculum sizes and to explore mechanisms of the inoculum effect individually and in combination, which would be challenging experimentally. We adopt a mathematical framework called the multi-hit model, which is based on the idea that antimicrobial molecules hit, i.e. bind to, cellular targets (33). In this approach, complex chemical reactions and molecular pathways are summarized in a simple mechanistic structure. This framework enables us to simulate bacterial population dynamics, from which we can infer the pharmacodynamic curve. We already used the multi-hit model in previous work (34) to describe the action of AMPs and a similar, albeit more antibiotic-specific, approach was used to model classic reaction kinetics of antibiotics (12).

A mechanistic explanation for the inoculum effect has already been provided by Abel zur Wiesch et al. (12). In their model, binding of molecules decreases the pool of free antimicrobials and thus the antimicrobial efficacy. Here, we use the multi-hit model to extend this analysis and apply the results to the PD function. With the multi-hit framework, we are able to ask how the changes in the inoculum size affects the pharmacokinetics of antimicrobials and how this in turn affects the PD curve. We show that reversible binding alone can explain the inoculum effect. Further, we quantify the inoculum effect in terms of change in PD parameter values with increasing inoculum 
size. In a next step, we analyze how the PD curve changes due to adding degradation of antimicrobial molecules by bacterial enzymes and illustrate how well the PD function predicts long-term population dynamics. Last, we apply our findings to the population dynamics of competing bacterial strains and predict how the inoculum size influences competition outcomes when a resistant strain is introduced.

\section{Results}

We developed a mathematical model to describe the interaction between a bacterial population and antimicrobials. The model is based on the multihit concept of antimicrobial action (33). In the model, bacteria are killed as soon as a certain number of antimicrobial molecules hit the cell. The modeling framework allowed us to keep track of the number of bacteria and antimicrobial molecules over time. We assumed that the interaction occurs in a constant volume. Hence, the amounts of bacteria and antimicrobial molecules are equivalent to their concentration.

\section{Model predictions on pharmacokinetics and pharmaco- dynamics}

First we used our multi-hit modeling framework to investigate if an inoculum effect emerges simply as a result of concentration-dependent interactions between bacteria and antimicrobials and their binding kinetics. To this end, 


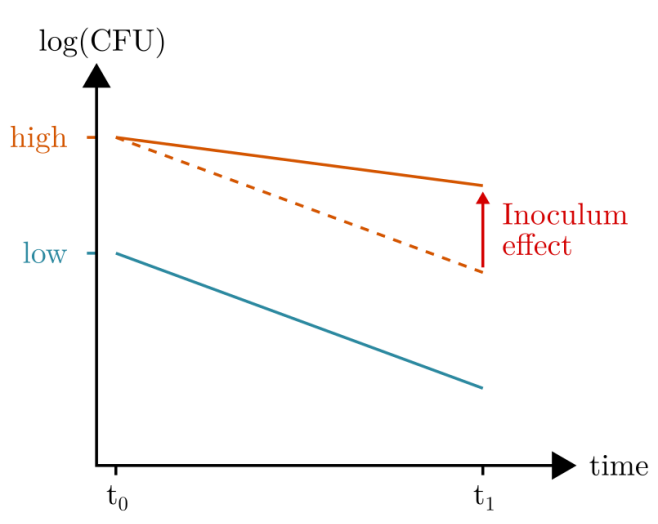

(a)

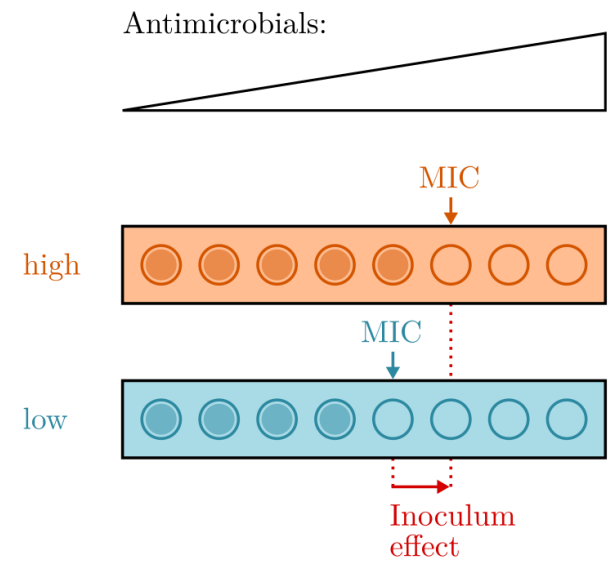

(b)

Figure 1: The inoculum effect changes efficacy of antimicrobials. Two common methods to quantify the efficacy of antimicrobials are (a) time-kill curves and (b) MIC measurements. (a) Time-kill curves illustrate the change in population size over time in presence of antimicrobials. The bacterial inoculum is the bacterial population size $N$ at $t_{0}=0 h\left(N_{\text {inoc }}=N(t=0)\right)$. The slope of the curve results from fitting a log-linear function to the population dynamics. The slope of time-kill curve representing dynamics of bacteria starting at a high inoculum size (orange) is higher than dynamics of bacteria starting at a low inoculum (blue). In the absence of an inoculum effect, the slope is independent of the inoculum (dashed orange line). (b) Alternative to measuring time-kill curves, efficacy is typically determined with the MIC. An inoculum effect is observed if increasing the inoculum size from low (blue) to high (orange) increases the MIC. 
our basic model assumed reversible binding of antimicrobial molecules to target sites on the bacterial cells. In the model, antimicrobials were recycled back into the system once a bacterial cell died. As a consequence, only the amount of free antimicrobials, but not their total amount, decreased during the time course.

The decrease in the amount of free antimicrobials was dependent on the bacterial inoculum $N_{\text {inoc }}$ (Fig. 2a). The higher the bacterial inoculum, the more antimicrobial molecules hit bacteria due to the mass-action type binding kinetics in our model, and the fewer antimicrobial molecules were free to hit in the following time step. The decrease in the amount of free antimicrobials due to initial binding to bacteria in the inoculum, in turn, changed the bacterial population dynamics. A bacterial population starting at a high bacterial inoculum grew over time, while a population exposed to the same amount of antimicrobials but starting at a low bacterial inoculum declined (Fig. 2b). Thus, binding of antimicrobial molecules - even though it was reversible - caused an inoculum effect, i.e. the antimicrobial decreased in efficacy with increasing bacterial inoculum. 
bioRxiv preprint doi: https://doi.org/10.1101/550368; this version posted May 12, 2020. The copyright holder for this preprint (which was not certified by peer review) is the author/funder, who has granted bioRxiv a license to display the preprint in perpetuity. It is made available under aCC-BY-NC-ND 4.0 International license.

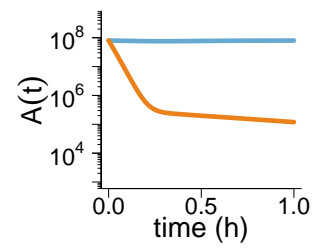

(a)

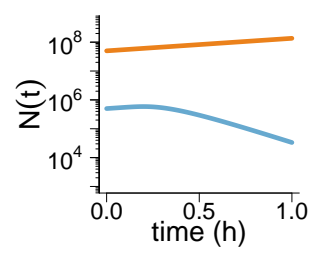

(b)

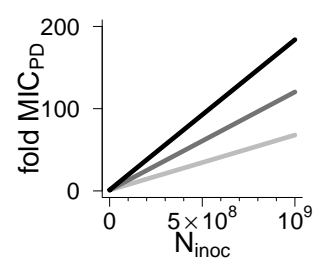

(d)

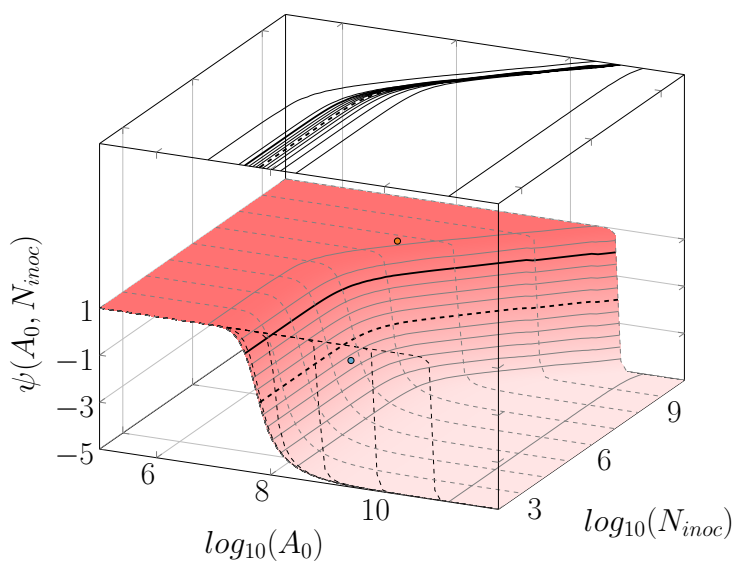

(c)

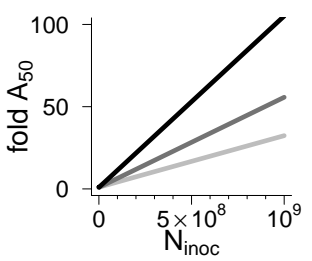

(e)

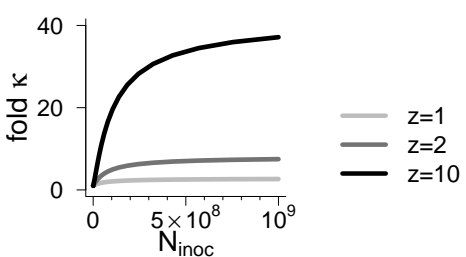

(f)

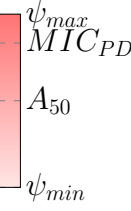

$A_{5}$$$
\text { (2) }
$$ 
Figure 2: Inoculum size affects pharmacokinetics and pharmacodynamics. PD functions are estimated for a range of inoculum sizes. Increasing the bacterial inoculum size from $5 * 10^{5}$ (blue) to $5 * 10^{7}$ (orange) changes the efficacy of antimicrobials (b) due to reversible reduction of the number of free antimicrobials (a). (c) We determined the efficacy for varying inoculum sizes $\left(N_{\text {inoc }}\right)$ with $z=10$, resulting in a PD plane (note that in contrast to (d)-(f), the inoculum effect here is illustrated on a $\log _{10}-\log _{10}$ scale). In the PD plane, solid gray contour lines show combinations of antimicrobials and inoculum sizes with the same net growth rate, both in the $\mathrm{PD}$ plane and projected above the PD plot. $M I C_{P D}$ and $A_{50}$ are indicated as bold solid and dashed line, respectively. For a number of inoculum sizes, we plotted the PD curve as dashed contour lines along the antimicrobial molecule number axis and again projected the contour lines, this time in front of the plot. The slopes of (b) are marked as dots in the corresponding colors. In (d)-(f) we plotted the PD parameters $M I C_{P D}, A_{50}$, and $\kappa$ against the bacterial inoculum for varying number of hits needed to kill a bacterial cell $(z)$. The bold black line in (d) corresponds to the bold solid line in (c) and the bold black line in (e) correspond the bold dashed lines in (c). Parameters not mentioned here are listed in Table S1. 
This inoculum effect altered the parameter of the pharmacodynamic (PD) function. The PD function describes the net growth rate of bacterial population for a given antimicrobial concentration and is a tool to describe efficacy of an antimicrobial. Here, we compared parameterized PD functions that are based on different inoculum sizes $N_{\text {inoc }}$ with the PD plane (Fig. 2c). The PD plane shows that the shape of the PD function changed with increasing in bacterial inoculum. To illustrate the magnitude of change, we plotted the net growth rates of the population dynamics pictured of Fig. 2b in the PD plane (Fig. 2c). The net growth rate of a population based on a small inoculum size (blue point) was negative, while it was positive for a populations based on a large inoculum (orange point). Note that both populations were exposed to the same amount of antimicrobials at the beginning of the simulation $\left(A_{0}\right)$. Without restricting $A_{0}$, the inoculum effect vanishes (Fig. S2). The example illustrates that the PD function and therefore the PD parameters are dependent on the bacterial inoculum.

In terms of specific $\mathrm{PD}$ parameters, both the $M I C_{P D}$, the amount of antimicrobials that results in zero growth, and $A_{50}$, the antimicrobial concentration that results in half of the maximum effect, increased with increasing inoculum (Fig. 2d, 2e). The increase was linear and increased for increasing number of targets needed to kill a cell ( $z$ ) (Fig. 2d, 2e). Superfluous binding according to the fractional single hit model, i.e. antimicrobials can hit more than $z$ targets, increased the inoculum effect, as well as nonreversible binding, and non-targeted binding combined with non-reversible 
binding (Fig. S3). The steepness of the curve, parameterized with the PD parameter $\kappa$, increased non-linearly with increasing inoculum size (Fig. 2f). The maximum effect of the antimicrobial $E_{\max }$ is defined by the parameters $\psi_{\max }$, the maximum growth rate in absence of the antimicrobial $\left(\psi_{\max }=\psi(A=0)\right)$, and $\psi_{\min }$, the minimum net growth rate for $A \rightarrow \infty$. In previous work (34) we showed that $\psi_{\max }$ is only dependent on replication and death, parameterized by the multi-hit parameter $b$ and $d$, respectively, with $\left(\psi_{\max }=b-d\right)$. We also showed that $\psi_{\min }$ is solely determined by the death rate of the zombie stages $\left(d_{z}\right)(34)$. This means, both PD parameters $\psi_{\max }$ and $\psi_{\min }$ and therefore also the parameter $E_{\max }$ were independent of any binding processes and the bacterial inoculum (Fig. S4b, S4c).

\section{Quantifying the inoculum effect}

We quantified the inoculum effect in terms of the PD function by incorporating how the PD parameters change due to changes in the bacterial inoculum. We already determined that both $M I C_{P D}$ and $A_{50}$ increased linearly with increasing bacterial inoculum in our basic multi-hit model. The linear increase is a direct measure of the inoculum effect and therefore, we used the linear relationship $A_{0, \psi}\left(N_{\text {inoc }}\right)=m N_{\text {inoc }}+c$ to quantify the inoculum effect. This linear relationship describes how many antimicrobial molecules $A_{0}$ we need to achieve an effect, specified in terms of net growth rate $\psi$, for a given bacterial inoculum size $N_{\text {inoc }}$. The slope $m$ in this relation measures the inoculum effect. The inoculum effect increased with increasing number 
of hits needed to kill a cell $(z)$, and was higher when we assumed superfluous binding, non-reversible binding, and non-reversible and non-targeted binding compared to the basic model of reversible binding (Table 1). Moreover, it was higher for $A_{50}$ than for $M I C_{P D}$ in all the above cases. While the intersection $c$ was influenced by the number of hits needed to kill a cell (Table 1) it is per definition independent of the inoculum effect. The slope $m$ and the intersection $c$ describe how many antimicrobial molecules we need to achieve a given pharmacodynamic efficacy. With these two parameters, we can also describe how many more antimicrobial molecules we need to achieve the same pharmacodynamic efficacy when we increase the bacterial inoculum $x$-fold:

$$
\frac{A_{0}\left(x N_{\text {inoc }}\right)}{A_{0}\left(N_{\text {inoc }}\right)}=\frac{m x N_{\text {inoc }}+c}{m N_{\text {inoc }}+c}<x \text { for } c>0
$$

Thus, the increase in antimicrobials required to achieve the same effect on an $\mathrm{x}$-fold increased inoculum is, according to our model, less than $\mathrm{x}$-fold.

The PD parameter $\kappa$ was calculated in terms of the PD parameters $M I C_{P D}$ and $A_{50}$. The bacterial inoculum size $N_{\text {inoc }}$ affected the shape and position of the pharmacodynamic curve, with the PD parameter $M I C_{P D}$, $A_{50}$ and $\kappa$. Temporary binding of antimicrobial molecules alone was enough to result in a perceivable inoculum effect and the inoculum effect was intensified if we assumed more complex binding processes. In agreement with this result, the inoculum effect was abrogated if the free antimicrobial concentration was assumed to be constant independent of any binding processes 
Table 1: Results of fitting linear function $x=m_{x} N_{\text {inoc }}+c_{x}$ with $x=M I C_{P D}$ and $x=A_{50}$ to the simulated data. Reversible binding with $z=1,2,10$ is also plotted in Fig. 2d - 2f.

\begin{tabular}{rrlccccc}
\hline & & & \multicolumn{2}{c}{$M I C_{P D}$} & & \multicolumn{2}{c}{$A_{50}$} \\
\cline { 7 - 8 } $\mathrm{z}$ & $\mathrm{n}$ & binding & $c_{M I C_{P D}}$ & $m_{M I C_{P D}}$ & & $c_{M I C_{P D}}$ & $m_{A_{50}}$ \\
\hline 1 & 1 & reversible & 2514516 & 0.167 & & 8284221 & 0.258 \\
2 & 2 & reversible & 6234471 & 0.728 & & 14665506 & 0.812 \\
10 & 10 & reversible & 37675418 & 6.508 & & 61533236 & 6.577 \\
10 & 39 & reversible & 37368958 & 6.672 & & 61618874 & 6.902 \\
10 & 10 & non-reversible & 36111621 & 9.081 & & 62435986 & 9.944 \\
10 & 10 & non-reversible, non-targeted & 57313826 & 9.416 & & 111901448 & 11.08 \\
\hline
\end{tabular}

206 (Fig. S2). Using our results, we were able to describe the net growth of 207 bacteria for given $N_{\text {inoc }}$ and $A$ :

$$
\begin{aligned}
& \psi\left(A_{0}, N_{\text {inoc }}\right)=\psi_{\max }-\frac{E_{\max }\left(\frac{A_{0}}{A 50\left(N_{\text {inoc }}\right)}\right)^{\kappa\left(N_{\text {inoc }}\right)}}{1+\left(\frac{A_{0}}{A 50\left(N_{\text {inoc }}\right)}\right)^{\kappa\left(N_{\text {inoc }}\right)}} \\
& =\psi_{\max }-\frac{\left(\psi_{\max }-\psi_{\min }\right){\frac{A_{0}}{M I C_{P D}\left(N_{\text {inoc }}\right)}}^{\kappa\left(N_{\text {inoc }}\right)}}{{\frac{A_{0}}{M I C_{P D}\left(N_{\text {inoc }}\right)}}^{\kappa\left(N_{\text {inoc }}\right)}-{\frac{\psi_{\min }}{\psi_{\max }}}}
\end{aligned}
$$

with

$$
\begin{aligned}
A_{50}\left(N_{\text {inoc }}\right) & =m_{A_{50}} N_{\text {inoc }}+c_{A_{50}} \\
M I C_{P D}\left(N_{\text {inoc }}\right) & =m_{M I C_{P D}} N_{\text {inoc }}+c_{M I C_{P D}} \\
\kappa\left(N_{\text {inoc }}\right) & =\frac{\log \left(-\frac{\psi \max }{\psi m i n}\right)}{\log \left(\frac{M I C_{P D}\left(N_{\text {inoc }}\right)}{A_{50}\left(N_{\text {inoc }}\right)}\right)}
\end{aligned}
$$




\section{Degradation enhances the inoculum effect}

Next, we extended our basic model to integrate proteolytic degradation of antimicrobials by extracellular enzymes. Bacteria that are able to express extracellular enzymes have often been used to explore the inoculum effect $(17,18,19,20,21,22)$. Assuming that each cell produces enzymes, the amount of enzymes is dependent on the bacterial density. In contrast to reversible binding, which we assumed in our basic model, degradation of antimicrobials decreased the number of antimicrobials irreversibly, i.e. the total number of antimicrobials in the system decreased.

Degradation of antimicrobial molecules in addition to reversible binding enhanced the effect the bacterial inoculum has on the dynamics of the antimicrobials. While in the basic model the amount of free antimicrobials was only reduced due to binding, degradation decreased the amount of free antimicrobials further (Fig. 3a). The reduction of antimicrobials in turn enabled higher bacterial population sizes (Fig. 3b) compared to the basic model. Not surprisingly, quantification of the inoculum effect based on PD parameters showed that the inoculum effect was stronger assuming degradation on top of reversible binding compared to reversible-binding only: The slopes of $M I C_{P D}$ and $A_{50}, m_{M I C_{P D}}$ and $m_{A_{50}}$, were higher compared to reversible binding (Fig. 3d, 3e). Again, due to the assumptions in the model, the size of the PD curve, i.e. parameter $\psi_{\max }, \psi_{\min }$, and therefore $E_{\max }$ were not affected by the inoculum (Fig. S5a, S5b). 


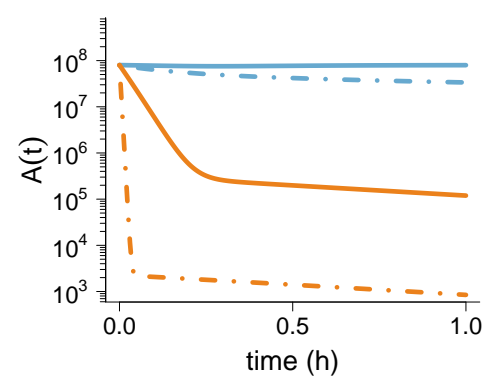

(a)

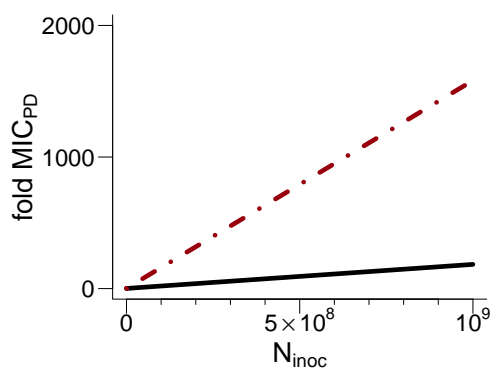

(d)

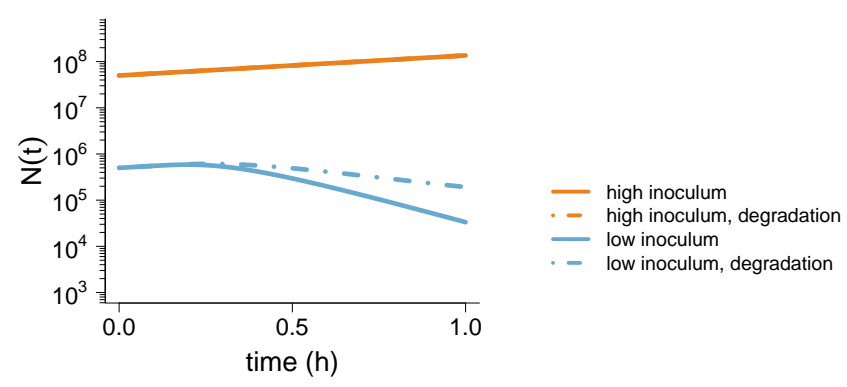

(b)

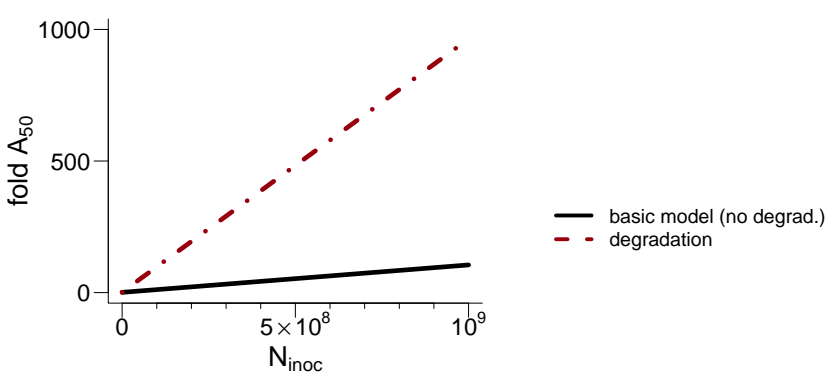

(e)

Figure 3: Degradation enhances the inoculum effect. (a) Antimicrobials are affected by degradation depending on the (b) bacterial population size. Here, we use two examples of bacterial inoculum sizes: low (blue) and high (orange). Changes in the dynamics of antimicrobials due to degradation depend on the initial bacterial inoculum size. In general, degradation (purple, dashed line) increases the number of molecules needed to achieve (c) $\psi\left(M I C_{P D}\right)=0$ and (d) $\psi\left(M I C_{A_{50}}\right)=E_{\max } / 2$ compared to reversible binding only (black line). All parameters used for the simulations are listed in Table S1. 


\section{Evaluation of the extended PD function}

The PD function $\psi\left(A_{0}\right)$ describes efficacy based on initial dynamics in the system and has been used to extrapolate long term bacterial population dynamics, e.g. $(1,13,35,36,37,38,39)$. Here, the underlying assumption is that the long-term bacterial population dynamics can be predicted on the basis of the initial short-term dynamics. This assumption is only valid if, at any time during the time-kill process, the net replication rate of a bacterial population of size $N$ can be approximated by $\psi\left(A_{0}, N\right)$ that has been derived from the initial decline. This may not be the case because at later times the bacterial cells will have bound antimicrobial molecules, resulting in a state more susceptible to the antimicrobial effect. For these reasons, we asked if the extended PD function $\psi\left(A_{0}, N_{\text {inoc }}\right)$ can be used to describe long term dynamics.

The multi-hit model enabled us to analyze if the short term population dynamics captured in the extended PD function predict long term dynamics. For this, we parameterized the extended PD function $\psi\left(A_{0}, N_{\text {inoc }}\right)$ based on short term population dynamics from the multi-hit model. The multi-hit was then extended with a carrying capacity to account for density dependent reduction in growth and used to simulate long term population dynamics. The long term population dynamics from the multi-hit model were compared to predictions from a simple population model that relies on the extended PD function.

Fig. 4 (solid lines) shows that the two approaches are in reasonable agree- 
ment assuming reversible binding only (basic model). Although the extended PD function underestimated the pharmacodynamic efficacy of the antimicrobial for low antimicrobial concentrations, the overall dynamical behavior of the system could be captured. In contrast, the dynamics based on the multihit model, in which enzymatic degradation was allowed, differed fundamentally from the dynamics predicted with the pharmacodynamic function at low bacterial inoculum (4, blue, dashed lines). Degradation decreased antimicrobials over time and the population was eventually able to increase in size. This effect was more pronounced at low bacterial inoculum sizes because at high bacterial inoculum sizes, the antimicrobial had already only little effect in the basic model. The extended PD function therefore captured simple bacterial population dynamics while only the mechanism-based multi-hit model could describe more complex population dynamics.

\section{Predicting the outcome of bacterial competition}

We used the multi-hit model to ask how the inoculum effect influences competition and, more specifically, how the bacterial inoculum affects the competitiveness of a recently mutated, more resistant strain. To answer this question, we extended the multi-hit framework to two bacterial strains, strain $S$ and $R$. Strain $R$ is more resistant to the antimicrobial than strain $S$ due to a 10 $\times$ lower attachment rate $\alpha$. Note that production of extracellular enzymes is also a mechanism of bacterial resistance, but here we tried to avoid complex population dynamics to be able to compare the results of the multi-hit 


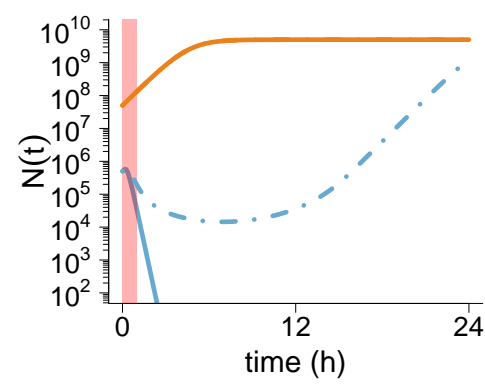

(a)

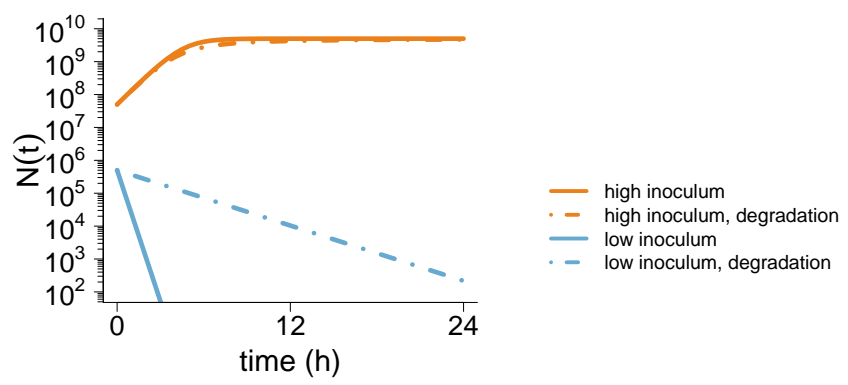

(b)

Figure 4: Dynamics of bacterial population $N(t)$ within the first $24 \mathrm{~h}$ after exposure to antimicrobials were simulated with (a) the multi-hit model and (b) a population model that incorporates $\psi\left(A_{0}, N_{\text {inoc }}\right)$ (equation 22$)$. (a) We compared dynamics without extracellular proteins (solid lines) and with extracellular proteins (dashed lines) as well as dynamics for low (blue) to high (orange) initial bacterial population sizes $N_{\text {inoc. }}$ For the dynamics of $A(t)$ and $E(t)$, see Fig. S6. The red area marks the time frame $(0 h \leq t \leq 1 h)$ used to determine the net growth rate $\psi\left(A_{0}, N_{\text {inoc }}\right)$, on which (b) is based on. For a higher resolution of this time frame, but without carrying capacity, see Fig. 3a, 3b. (b) We predicted long term dynamics based on $\psi\left(A_{0}, N_{\text {inoc }}\right)$ with a simple population model (equation 22). All parameters used for the simulations are listed in Table S1. 
model with the dynamics based on the PD function. By extending the basic model by a strain $R$, we were able to simulate competition under complex pharmacokinetics (PKs).

We were interested if a resistant strain that is introduced in the system can take over the population within the simulation time. Strain $R$ was not able to outcompete strain $S$ at low antimicrobial concentrations (Fig. 5a) due to an implemented cost of resistance. At intermediate concentrations, $R$ took over the population (Fig. 5b) and at high concentrations, both strains were killed (Fig. 5c). These examples illustrate that the outcome of competition depended on antimicrobial dose given $\left(A_{0}\right)$ and we asked how both antimicrobial concentration at the beginning of the simulation and bacterial inoculum size change the outcome of competition.

We therefore extended our analysis for a broad spectrum of antimicrobial input concentrations and bacterial inoculum sizes (Fig. 5d). Here the range of antimicrobial concentrations for which the resistant strain can take over the population was dependent on the bacterial inoculum $N_{\text {inoc }}$. The higher the bacterial inoculum the smaller was the range of antimicrobial concentrations in which the resistant strain took over. These results were qualitatively reproduced with the extended PD function (Fig. S7).

For comparison, we simulated the competition between the two strains in a model that does not display the inoculum effect. To parameterize such a model, we determined the PD function $\psi\left(A_{0}\right)$ for $N_{\text {inoc }}=5 \times 10^{5}$. As expected, without the inoculum effect, the competitiveness of $R$ did not 

aCC-BY-NC-ND 4.0 International license.

299 depend on the inoculum (Fig. 5e), and was overestimated, especially at high 300 inoculum sizes (compare Fig. 5e and Fig. 5d). 


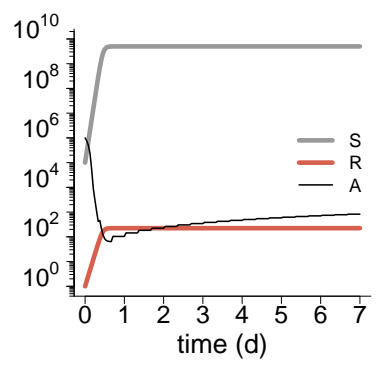

(a)

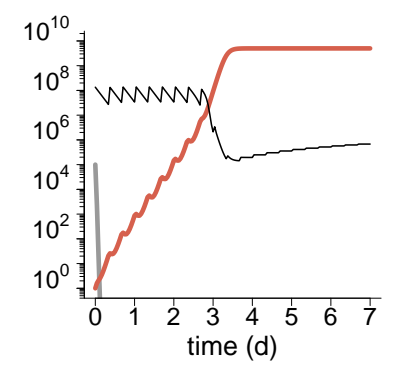

(b)

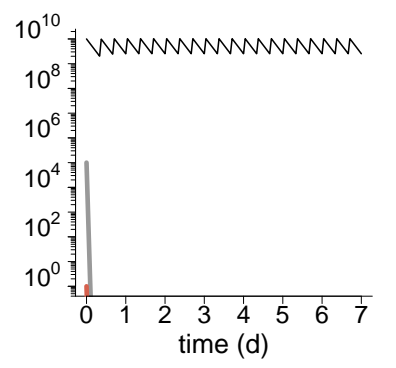

(c)

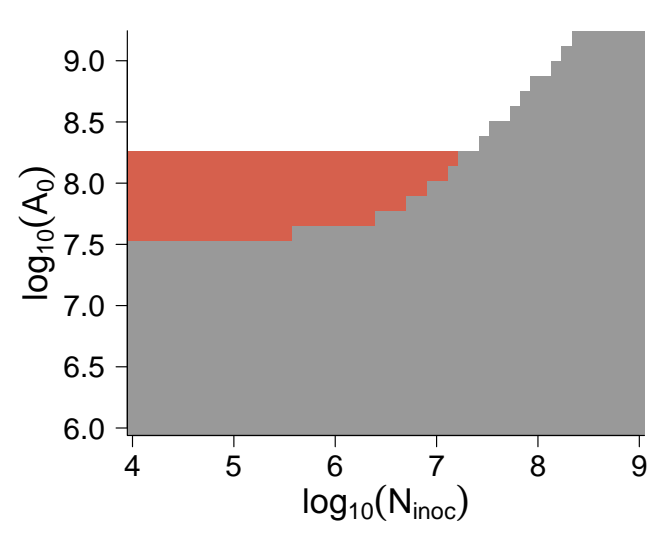

(d)

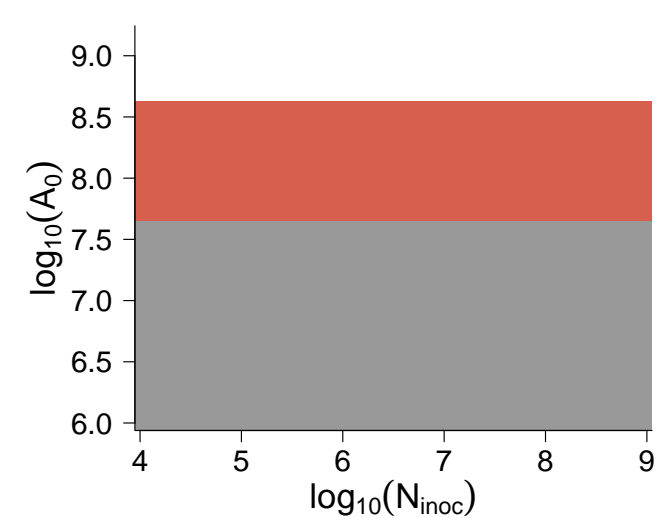

(e)

Figure 5: The inoculum effect influenced competition outcomes. Antimicrobial doses $A_{0}$ changed the outcome of competition: (a) $S$ won when exposed to low antimicrobial doses, (b) $R$ won at intermediate antimicrobial doses and (c) bacteria went extinct at high antimicrobial doses. (d) In an additional dimension, the inoculum effect changed the outcome of competition (gray: $S$ is more abundant, red: $R$ is more abundant, white: extinction $(S, R<1)$ at the end of the simulation). (e) For comparison, we used the PD function to simulate competition without inoculum effect. 
301

\section{Discussion}

The inoculum effect has been demonstrated experimentally for a number of bacterial strains and antimicrobials $(13,17,18,21,22,26,27,29,30,40,41)$. In most cases the MIC $(13,17,22,26,27,29)$ was used to determine and quantify the inoculum effect. Udekwu et al. (13) demonstrated how to use MIC measurements to integrate the inoculum effect as change in $M I C_{P D}$ in the PD function. However, they assumed that other PD parameters, among other things $\kappa$, were not affected by the inoculum effect.

In this work, we analyzed the inoculum effect based on mathematical modeling of bacterial population dynamics. We investigated whether the bacterial inoculum size has an impact on the efficacy of antimicrobials as measured by the PD function. A mechanism-based mathematical framework called multi-hit model (33) enabled us to explore the effect of increasing the inoculum size. Our findings are consistent with Abel zur Wiesch et al. (12): due to the kinetics of antimicrobials binding to bacterial cells, larger amounts of antimicrobials are required to attain the same efficacy when the bacterial inoculum size is increased.

We examined this inoculum effect quantitatively: the inoculum affected the PD parameters $M I C_{P D}, A_{50}$, and $\kappa$. We showed that a change in efficacy cannot only be achieved by varying the antimicrobial concentration, but also achieved by varying the bacterial inoculum. Formally, the $M I C_{P D}, A_{50}$, and $\kappa$ increased with increasing inoculum size. Consequences of the inoculum ef- 
fect may be altered outcome of survival and resistance evolution experiments, which we have previously shown are dependent on the PD parameters (37).

In our basic model, the reason for the inoculum effect is that an increase in the bacterial inoculum results in higher depletion in the pool of free antimicrobials, we call this a "numbers game": Binding, even if it is reversible, takes molecules out of the pool of free antimicrobial molecules, which in turn means that there are fewer molecules available for binding in the next time step, This numbers game has already been observed with mathematical modeling $(12,33)$, however the experimental validation of the inoculum effect based on the numbers game alone is sparse $(12,13,30)$ with the focus being mainly on enzymatic degradation (for an overview, see (15)). We argue that studies that experimentally measure the inoculum effect based on the numbers game are vital for fully understanding the impact of the inoculum effect.

Moreover, not only the numbers game in itself but also how variations of the numbers game affect the efficacy of antimicrobials should be tested experimentally. For example, our simulations show that the more targets are needed to be hit to kill a cell $(z)$, the more pronounced is the inoculum effect on the PD parameters $M I C_{P D}, A_{50}$, and $\kappa$. This result suggest that the inoculum effect is stronger the more antimicrobial molecules are needed to kill a cell. In the case of antimicrobial peptides, this number varies from 80800 in the case of protegrin (42) to $10^{6}-10^{7}$ in the case of PMAP-23 (43). With everything else being equal, we therefore expect a more pronounced 
inoculum effect for PMAP-23 than for protegrin.

In a next step, we determined the effect of enzymatic degradation in addition to the binding effects. The inoculum effect is often measured experimentally in systems with enzymes present that degrade antimicrobial molecules, for example $\beta$-lactamases $(17,18,21,22,26,27,40,41,44)$. When allowing for enzymatic degradation in our simulations, the inoculum effect, quantified as increase in the pharmacodynamic parameters $M I C_{P D}$ and $A_{50}$, was more pronounced. In these simulations, bacterial dynamics also increased in complexity, in particular at low bacterial concentrations, which influenced the accuracy of the extended PD function $\psi\left(A, N_{\text {inoc }}\right)$ to predict the bacterial population dynamics.

Similar to the effects of enzymatic degradation, non-targeted binding enhanced the inoculum effect in our simulations. Non-targeted binding was implemented as binding to intracellular components of dead cells. Dead cells were assumed to leak intracellular content that posed alternative targets for antimicrobial molecules. As a result, the inoculum effect was more pronounced: the increase of the pharmacodynamic parameters $M I C_{P D}$ and $A_{50}$ was steeper with non-targeted binding than in the basic model. In accordance with our modeling results, experiments showed that non-targeted binding decreased the amount of antimicrobial peptide molecules available to kill bacteria (45).

To investigate the influence of the inoculum effect on bacterial competition, we extended the basic multi-hit model by a resistant strain $R$. We mod- 
eled resistance as a decrease in the hit rate $\alpha$, which corresponds to resistance due to target modification (23). Simulation of bacterial competition revealed that the competitiveness of the resistant strain is overestimated when the inoculum effect is not considered. The range of antimicrobial concentration, in which $R$ can take over is a rough estimate of the mutant selection window (MSW) $(46,47)$, which we call "realized MSW". The difference between the MSW and the realized MSW is that the MSW is based on time-kill curves with a single input of antimicrobials, while the realized MSW is based on time-kill curves in which the antimicrobials are repeatedly inputted. In our simulations, the estimated MSW becomes smaller for higher bacterial inoculum sizes. This means that, while a higher population size of the sensitive strain $S$ increases the probability of resistance emergence, more sensitive bacteria in the presence of antimicrobials also decreases the competitiveness of the emerged resistant strain by reducing antimicrobial efficacy. We expect the selection coefficient in competition experiment, e.g. (47), to decrease with increasing inoculum. Moreover, this unexpected feature of the inoculum effect might be of relevance for epidemiological problems, for example co-existence of drug resistant and sensitive bacteria $(48,49)$.

The absence of the inoculum effect has been reported repeatedly $(19,50)$. In our system, the inoculum effect was not perceivable in certain regions of the PD plane: for very small and very high antimicrobial numbers, the inoculum effect was absent. In addition, the inoculum effect on the MIC might be challenging to detect because of inherent uncertainties in determining the 
MIC by using doubling dilutions. As a concrete example, if, by increasing the inoculum, the "real" value of the MIC increases from 9 to $15 \mu \mathrm{g} / \mathrm{ml}$, the measured MIC would be in both cases be $16 \mu \mathrm{g} / \mathrm{ml}$. Furthermore, in many studies an arbitrary threshold of an 8-fold increase of the MIC for a 100-fold higher bacterial inoculum is used to define the inoculum effect with MIC measurements $(9,17,18,21,22)$, clouding inoculum effects of smaller extent. More precise determination of the bacterial population size, e.g. with flow cytometry, could help to precisely quantify the inoculum effect.

In addition, it has been argued that the inoculum effect is an artifact (19). The core of this argument is that, to obtain the same pharmacodynamic efficacy, increasing the bacterial inoculum requires a proportionate increase in the amount of antimicrobials. Following this line of thought, keeping the ratio of antimicrobials to bacteria $\left(\frac{A_{0}}{N_{\text {inoc }}}\right)$ constant should always result in the same pharmacodynamic efficacy. In our simulations, however, the ratio of antimicrobials to bacteria $\left(\frac{A_{0}}{N_{\text {inoc }}}\right)$ does not predict efficacy. Rather, to obtain the same effect on a 100-fold increased bacterial population, we require less than 100-fold increase in antimicrobial molecules. For example, if we increase the bacterial inoculum from $5 \times 10^{5}$ to $5 \times 10^{7}$, we only need a 9 -fold increase in antimicrobial molecules to achieve the same pharmacodynamic efficacy and the ratio $\frac{A_{0}}{N_{\text {inoc }}}$ decreases from 80 to $7^{1}$.Thus, with increasing inoculum, each antimicrobial molecule becomes actually more effective.

\footnotetext{
${ }^{1}$ Calculations are based on equation 1 and parameter values of slope $m$ and intersection $c$ are listed in Table 1 (reversible binding, $n=z=10$ ).
} 
The scale of the inoculum effect depends on the binding mechanisms of the antimicrobial, the target hit number $z$, and of the effect size of the antimicrobial that we are interested in. The latter one gives us an clue where to best measure the inoculum effect: The PD plane (Fig. 2c) shows that the inoculum effect is best measured at concentrations close to the $A_{50}$, because at low and very high concentrations, we do not expect to see an inoculum effect. More precisely, our quantitative analysis shows that $\kappa$ increases, which means that the inoculum effect is stronger for $A_{50}$ than for $M I C_{P D}$. This means that the effect of $A_{50}$ is most suitable to measure the inoculum effect. Based on this observation, we recommend to not only quantify the $M I C$, but the whole PD function curve and to use optical density (OD) measurements with caution, since with OD measurements, only concentrations up to the $M I C_{P D}$ can be measured.

In summary, we investigated the effect of the bacterial inoculum on the efficacy of antimicrobials in a simple conceptual model that describes the attachment and detachment dynamics of antimicrobial molecules to target sites on the bacteria. We showed that the inoculum effect emerges from these simple dynamics. The quantitative aspect of this effect are surprisingly complex. In our mechanistic population model, the inoculum effect was caused solely by binding and degradation, but further mechanisms have been invoked to explain the inoculum effect. The efficacy of antimicrobials can change because of bacterial density dependent change of abiotic factors. For example, pH (31), accessibility (23), susceptibility to antimicrobials $(32,51)$ 
can all change with bacterial density. These factors likely play an important role in vivo, however studying the inoculum effect focuses mainly on empf yet remains to be explored, with a few notable exceptions (23). Although we did not focus on these mechanisms, it is possible to extend the multihit framework to incorporate these explanations. These further mechanisms will exacerbate the inoculum effect that arises from the binding kinetics. We also showed that the inoculum effect influences the competitive dynamics between bacterial strains under treatment. We conclude that a comprehensive quantitative description of pharmacodynamics requires a detailed mechanistic population model and cannot be captured by simple pharmacodynamic functions $(1,13,37,39)$.

\section{Methods}

\section{Multi-hit model}

We used the multi-hit model introduced by Hedges (33) to explore bacterial population dynamics in silico in presence of antimicrobial peptides (AMPs). We have already applied a modified version of the multi-hit model in previous work (34) and a similar approach has been introduced by Abel zur Wiesch et al. (12) to describe effects of antibiotics.

The multi-hit model describes a bacterial population consisting of $N_{t o t}$ cells. In the presence of antimicrobials, bacterial cells are hit by antimicrobial molecules. The key idea of this population model is to categorize all 
bacterial cells according to how many antimicorbials have hit the cell (Fig. 6). A hit is the mathematical simplification of adsorption and subsequent downstream processes connected to binding of one antimicrobial molecule to a bacterial target site and is described with the rate $\alpha$. The parameter $\mu$ is the detachment of an antimicrobial from the bacterial target. Bacteria that have $i$ antimicrobial molecules adsorbed are categorized in class $N_{i}$. The number of absorbed molecules varies from cell to cell, with $0 \leq i \leq n$. Thus, we have a total of $n+1$ classes and $N_{t o t}=\sum_{i=0}^{n} N_{i}$. The rate of change of each subpopulation is described with the following ordinary differential equation:

$$
\begin{aligned}
\frac{d N_{i}}{d t}= & \alpha_{i-1} A N_{i-1}-\alpha_{i} A N_{i}+ \\
& \mu_{i+1}(i+1) N_{i+1}-\mu_{i} i N_{i}+ \\
& \Omega\left(N_{i}\right)-d_{i} N_{i}
\end{aligned}
$$

Each class $N_{i}$ gains bacterial cells from the class $N_{i-1}$ with the term $\alpha_{i-1} A N_{i-1}$. Here, we assumed that finding the bacterial cell is the rate limiting step for an antimicrobial molecule (52). This is appropriate if the number of targets are in abundance, e.g. when antimicrobial peptides adsorb to the cell membrane of bacterial cells. For antimicrobials that act within the cell, a hit rate dependent on the number of free targets is more appropriate (see (12)).

The term $\Omega\left(N_{i}\right)$ in equation 7 summarizes replication processes, which we modeled according to Abel zur Wiesch et al. (12). In short, cells of class $N_{i}$ replicate with the replication rate $b_{i}$. During replication, $x$ bound antimi- 
crobial molecules of the mother cells are redistributed to the two daughter cells according to a hypergeometric distribution:

$$
\Omega\left(N_{i}\right)=2 \sum_{x=i}^{n}\left(f_{x, i} b_{x} N_{x}\right)-b_{i} N_{i}
$$

The parameter $f_{x, i}$ denotes the fraction of cells from class $N_{x}$ that are redistributed to $N_{i}$ due to replication.

Bacteria in class $N_{i}$ die with a natural death rate $d_{i}$ and due to interaction with the antimicrobial. We assume that the antimicrobial is bactericidal only. The bactericidal effect becomes apparent in the zombie stage. Once a threshold number of antimicrobial molecules has hit the cell $(i=z)$, the cell enters the zombie class. In the basic model, we assumed complete multi-hit (33), with $z=n$. In the zombie class, bacteria are doomed to die and do so with an increased death rate $d_{z}>d$.

The multi-hit model allows us to keep track of the amount of antimicrobials $A$. In the basic model, there is no loss of antimicrobials due to nonreversible binding, non-targeted binding, or degradation, i.e. we assumed that all antimicrobial molecules bound to a bacterium are recycled back into the system after the death of this cell:

$$
\frac{d A}{d t}=\sum_{i=0}^{n}\left(\mu_{i} i N_{i}+d_{i} i N_{i}-\alpha_{i} A N_{i}\right)
$$

Here, the variables $N$ and $A$ are used in a term describing mass action kinetics. Therefore, $N$ and $A$ are typically concentrations of bacteria and 
antimicrobial molecules, respectively (53). In the framework of the multi-hit model, it is however necessary to use the absolute amount of bacteria and antimicrobial molecules because we keep track of how many antimicrobial molecules have hit the bacterial cells (12). The volume of the system $V$ is assumed constant, with $V=1 \mathrm{ml}$. Resulting, the number of bacteria cells and antimicrobial molecules are equivalent to their concentration values.

\section{Variations in the Multi-hit framework}

We determined the multi-hit model based on the ODE system presented above. In the basic model (equation 7-9), we assumed the case of complete multi-hit (33), with $z=n$. The complete multi-hit model is appropriate for antimicrobials that need to hit a large fraction of the existing targets. An example of such a case are AMPs that kill according to the carpet model (43). Moreover we assumed that the total amount of antimicrobials is stable and that antimicrobial molecules are recycled back into the system when a cell dies. We relaxed these assumptions with (i) superfluous binding, (ii) non-reversible binding, (iii) non-targeted and non-reversible binding, and (iv) enzymatic degradation.

In the case of (i) superfluous binding, we implemented the fractional multi-hit model by Hedges (33). In this model, $z<n$, i.e. binding to targets is possible although the cell already reached the zombie stage. The fractional multi-hit model describes cases like AMPs that kill by forming pores into the membrane of bacteria (42). Here, a cell has more targets, i.e. places where 


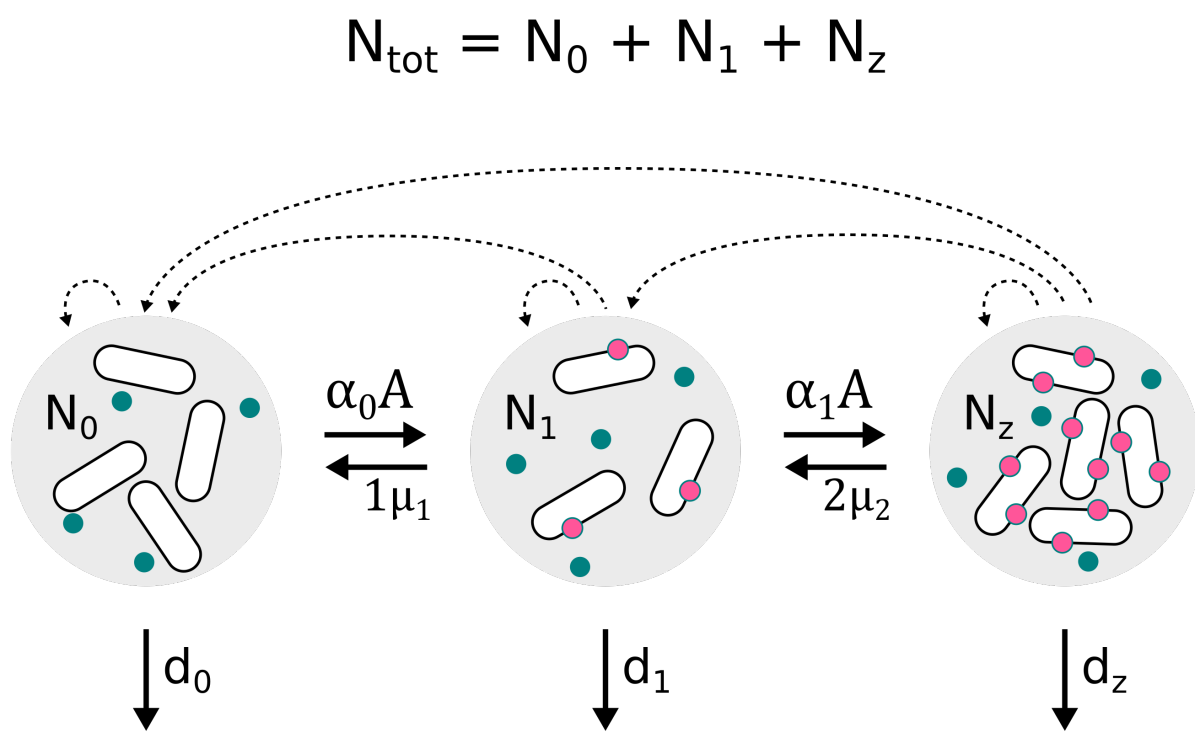

Figure 6: Schematic representation of the basic multi-hit model with three classes $(n=2)$. At every time point the bacterial population $N_{\text {tot }}$ can be divided in $n$ subpopulations based on the number of antimicrobials that have hit the bacterial cells. In this example, a bacterium dies with an increased death rate $d_{z}$ if two antimicrobial molecules have hit the cell, i.e. $z=2$. Therefore, $N_{t o t}=N_{0}+N_{1}+$ $N_{2}$, with $N_{2}=N_{z}$. Antimicrobials that have hit a cell are indicated in pink. $A$ is the number of free antimicrobial molecules, here indicated in blue. The rates of the ODE (equation 7) are indicated with arrows. For simplicity, bacterial replication according to equation 7,8 is only indicated with dashed arrows. 
a pore can be formed in the membrane, than pores needed to kill a cell.

In the case of (ii) non-reversible binding, we set $\mu=0$ and assumed that antimicrobial molecules are not recycled back into the system. Examples of non-reversible binding are binding of the antibiotics streptomycin and kanamycin to bacterial ribosomes (54). This results in

$$
\frac{d A}{d t}=\sum_{i=0}^{n}-\alpha_{i} A N_{i}
$$

In the case of (iii) non-reversible and non-targeted binding, we assumed that in addition to non-reversible binding, molecules attach irreversibly to leaked cell content of dead cells. In our model, dead cells leak $l$ targets per dying cell for non-targeted binding. $L$ is the total number of targets available for non-targeted binding with AMPs with the rate $\alpha_{d}$.

$$
\begin{aligned}
& \frac{d L}{d t}=l \sum_{i=0}^{n} d_{i} N_{i} \\
& \frac{d A}{d t}=\sum_{i=0}^{n}\left(-\alpha_{i} A N_{i}-\alpha_{d} A L\right)
\end{aligned}
$$

As in the cases (ii) and (iii), enzymatic degradation (case (iv)) decreases the amount of antimicrobials in the system. Bacteria can for example produce proteases, e.g. aureolysin (25), which catalyze hydrolysis of AMPs. We assumed that all bacterial cells produce enzymes $E$ with the production rate 
$p$ and that enzymes degrade antimicrobial molecules with the rate $d_{E}$ :

$$
\frac{d E}{d t}=p \sum_{i=0}^{n} N_{i}-d_{E} E
$$

530 ${ }_{538}\left(A(t=0)=A_{0}\right)$, with

$$
\begin{aligned}
\frac{d E}{d t} & =p \sum_{i=0}^{n} N_{i}-d_{E} E-r_{1} E A+r_{2} E . A+r_{3} E . A \\
\frac{d E . A}{d t} & =r_{1} E A-r_{2} E . A-r_{3} E . A \\
\frac{d A}{d t} & =\sum_{i=0}^{n}\left(\mu_{i} i N_{i}+d_{i} i N_{i}-\alpha_{i} A N_{i}\right)-r_{1} A E+r_{2} E . A
\end{aligned}
$$

\section{Pharmacodynamic function}

$$
\begin{aligned}
& \psi\left(A_{0}\right)=\psi_{\max }-e\left(A_{0}\right) \\
& \psi\left(A_{0}\right)=\psi_{\max }-\frac{\left(\psi_{\max }-\psi_{\min }\right)\left(A_{0} / M I C_{P D}\right)^{\kappa}}{\left(A_{0} / M I C_{P D}\right)^{\kappa}-\psi_{\min } / \psi_{\max }}
\end{aligned}
$$


Here, the effect $e$ describes the reduction of the net growth rate due to antimicrobial $A_{0}$. The $M I C_{P D}$ is the antimicrobial concentration $A_{0}$ which results in a net growth rate of zero: $\psi\left(A_{0}=M I C_{P D}\right)=0$. Note that the $M I C_{P D}$ is a approximation of the $M I C$ if the antimicrobial is concentration independent (8). Other parameters of the PD function are the maximum net growth rate in absence of a drug $\psi_{\max }=\psi\left(A_{0}=0\right)$, the minimum net growth rate $\psi_{\min }=\psi\left(A_{0} \rightarrow \infty\right)$, and the slope parameter $\kappa$. Alternatively, the PD function is described with the parameters $\psi_{\max }$, the maximum effect $E_{\max }$, with $E_{\max }=\psi_{\max }-\psi_{\min }$, the dose that results in half of the maximum effect $A_{50}$, with $\psi\left(A_{50}\right)=E_{\max } / 2$, and $\kappa(1)$.

Together, the two definitions of the PD function allowed us to calculate the slope parameter $\kappa$ based on estimations of $\psi_{\max }, \psi_{\min }, M I C_{P D}$, and $A_{50}$, with

$$
\kappa=\frac{\log \left(-\frac{\psi \max }{\psi \min }\right)}{\log \left(\frac{M I C_{P D}}{A_{50}}\right)}
$$

To estimate the PD parameter, we simulated time-kill curves for which we varied the antimicrobial concentration (Fig. S1). In a first step, the net growth rate was calculated based on the simulated time-kill curves, with

$$
\psi(A)=\frac{\log \left(N_{t o t}\left(t_{1}\right)\right)-\log \left(N_{t o t}\left(t_{0}\right)\right)}{t_{1}-t_{0}}
$$


we determined $\psi\left(A_{0}=0\right)$ to estimate $\psi_{\max }$ and $\psi\left(A_{0}=10^{15}\right)$ to estimate $\psi_{\min } . E_{\max }$ was calculated as $\psi_{\max }-\psi_{\min }$. The expressions $\psi_{\max }-\frac{E_{\max }}{2}=$ $\psi\left(A_{0}=x\right)$ and $0=\psi\left(A_{0}=y\right)$ were optimized for $x$ and $y$ to parameterize $A_{50}$ and $M I C_{P D}$, respectively. Finally, we calculated $\kappa$ according to equation 18 .

Multiple such parameterized PD functions for varying bacterial inoculum sizes were used to ascertain the change in PD parameters due change in the bacterial inoculum. These changes in parameters were implemented in an extended the PD function, $\psi\left(A_{0}, N_{\text {inoc }}\right)$. The inoculum effect was quantified as slope $m$ of the function

$$
A_{0, \psi=y}\left(N_{\text {inoc }}\right)=m N_{\text {inoc }}+c,
$$

with $y=0$ and $y=E_{\max } / 2$ in the case of $\psi\left(A_{0}=M I C_{P D}\right)$ and $\psi\left(A_{0}=A_{50}\right)$, respectively.

\section{Pharmacokinetic function}

To model bacterial population dynamics over longer time periods, we used the pharmacokinetic (PK) function as described in Regoes et al. (1). In short, antimicrobials are in the system with the concentration $A_{0}$. Antimicrobial molecules decay with the decay rate $k_{A}$. Without binding, the pharmacokinetic $(\mathrm{PK})$ function is

$$
A(t)=A_{0} e^{k_{A}(t-p)}
$$


${ }_{573}$ with $n \times 8 h \leq t \leq(n+1) \times 8 h$.

\section{${ }_{574}$ Population model}

${ }_{575}$ The PD function and the pharmacokinetic function were implemented in a

576 population model to estimate population dynamics over time, with

$$
\frac{d N_{t o t}}{d t}=\psi_{\max } N\left(1-\frac{N}{K}\right)-e\left(A_{0}, N_{\text {inoc }}\right) N
$$

577 Here, $K$ is the carrying capacity and $\psi_{\max }$ is used as an estimate of the 578 replication rate. We used this framework before in (37). 
579

\section{Competition}

To describe competition between a sensitive strain $S$ and a resistant strain $R$, we extend the basic multi-hit model, with

$$
\begin{aligned}
\frac{d S_{i}}{d t}= & \alpha_{i-1} A S_{i-1}-\alpha_{i} A S_{i}+ \\
& \mu_{i+1}(i+1) S_{i+1}-\mu_{i} i N_{i}+ \\
& \Omega\left(S_{i}\right)-d_{i} S_{i} \\
\frac{d R_{j}}{d t}= & \alpha_{j-1} A R_{j-1}-\alpha_{j} A R_{j}+ \\
& \mu_{j+1}(j+1) R_{j+1}-\mu_{j} j R_{j}+ \\
& \Omega\left(R_{j}\right)-d_{j} R_{j} \\
\frac{d A}{d t}= & \sum_{i=0}^{n}\left(\mu_{i} i S_{i}+d_{i} i S_{i}-\alpha_{i} A S_{i}\right)+ \\
& \sum_{j=0}^{n}\left(\mu_{j} i R_{j}+d_{j} j R_{j}-\alpha_{i} A R_{i}\right)
\end{aligned}
$$

Here, $i$ and $j$ describe the number of targets hit on a sensitive and resistant bacterial cell, respectively, with $0 \leq i, j \leq n$. In our simulations, strain $R$ had a 10 times lower attachment rate $\left(\alpha_{j}=\alpha_{i} / 10\right)$ which reflects modification in the surface of bacterial cells. This is a common resistance mechanism against AMPs (23). To model realistic long term population dynamics, we also included a carrying capacity, as it is done in (12):

$$
\Omega\left(N_{i}\right)=\left(2 \sum_{x=i}^{n}\left(f_{x, i} b_{x} N_{x}\right)-b_{i} N_{i}\right)\left(1-\frac{N_{t o t}}{K}\right)
$$


${ }_{588}$ We simulated population dynamics in a window of 7 days.

\section{${ }_{589}$ Implementation}

590 The multi-hit model was implemented in R (55) (www.r-project.org; version 3.4.0) in RStudio (56) (version 1.0.143). We solved the deterministic multi-

592 hit model numerically with the package deSolve (57). For plotting, we used 593 the following packages: sfsmisc (58) and RColorBrewer (59). The code is 594 available upon request.

\section{${ }_{595}$ Authors contributions}

R.R.R. and D.Y.B. initiated this project, conceived the model, analyzed the results and drafted the manuscript. D.Y.B. wrote the code in R.

\section{Competing interests}

We have no competing interests.

\section{Funding}

R.R.R. acknowledges the financial support of the ETH Zurich (ETH-41 15-2). 


\section{${ }_{602}$ Acknowledgments}

${ }_{603}$ We thank Andrew Read for sparking our interest in the topic of inoculum ef-

604 fects, Pia Abel zur Wiesch, Sebastian Bonhoeffer, and Jens Rolff for reading

605 the manuscript, Bruce Levin for giving us valuable feedback on our discus-

606 sion, and João Pires for interesting discussions about the project. 
bioRxiv preprint doi: https://doi.org/10.1101/550368; this version posted May 12, 2020. The copyright holder for this preprint (which was not certified by peer review) is the author/funder, who has granted bioRxiv a license to display the preprint in perpetuity. It is made available under aCC-BY-NC-ND 4.0 International license.

\section{${ }_{607}$ Appendix}


Table S1: Variables, indices and parameters of the multi-hit model. If not men-

tioned elsewhere, the parameter values used for the simulations are listed here. All rates are in $\frac{1}{h}$.

\begin{tabular}{ll}
\hline Variables & Definition \\
\hline$N_{i}$ & Number of bacteria that are hit by $i$ antimicrobial molecules at time $t$. \\
$N_{t o t}$ & Total number of bacteria at time $t$, with $N_{t o t}=\sum_{i=0}^{n} N_{i}$. \\
$N_{\text {inoc }}$ & Number of bacteria at time $t=0$, with $N_{\text {inoc }}=N_{t o t}(t=0)$. \\
$A$ & Number of free antimicrobial molecules at time $t$, with $A_{0}=A(t=0)$. \\
$E$ & Number of extracellular bacterial enzyme molecules at time $t$. \\
& We used $E(t=0)=N_{\text {inoc }} p /\left(b_{0}-d_{0}-d_{E}\right)$. \\
$E . A$ & Number of enzyme-antimicrobial complexes at time $t$, with $E \cdot A(t=0)=0$. \\
$S$ & Number of sensitive bacterial cells at time $t$. \\
$R$ & Number of resistant bacterial cells at time $t$, with $R(t=0)=1$. \\
$L$ & Number of targets for non-targeted binding at time $t$, with $L(t=0)=0$.
\end{tabular}

\begin{tabular}{ll}
\hline Indices & Definition \\
\hline $\mathrm{n}$ & Total number of possible hits. \\
$\mathrm{i}, \mathrm{j}$ & Number of hits, with $0 \leq i, j \leq n$. For the simulations, $n=10$ \\
$\mathrm{z}$ & Number of hits needed to kill a bacterial cell, with $1 \leq z \leq n$. \\
& If not mentioned, $z=10$ is used.
\end{tabular}

\begin{tabular}{|c|c|c|}
\hline Parameters & Definition & Value \\
\hline$t$ & $\begin{array}{l}\text { time-point of the simulation, if not mentioned, } t_{0}= \\
0 h \text { and } t_{1}=1 h \text { are used to determine the net growth } \\
\text { rate of the bacterial population. }\end{array}$ & \\
\hline$b_{i}$ & $\begin{array}{l}\text { Replication rate of bacterial cells in class } N_{i} \text {, with } \\
i<z \text {. }\end{array}$ & 1 \\
\hline$b_{z}$ & $\begin{array}{l}\text { Replication rate of bacterial cells in class } N_{i} \text {, with } \\
i \geq z \text {. }\end{array}$ & 0 \\
\hline$d_{i}$ & Death rate of bacterial cells in class $N_{i}$, with $i<z$ & 0 \\
\hline$d_{z}$ & Death rate of bacterial cells in class $N_{i}$, with $i \geq z$. & 5 \\
\hline$\alpha_{i}$ & Hit rate of antimicrobial molecules with $0 \leq i \leq n$ & $5 \times 10^{-7}$ \\
\hline$\alpha_{n}$ & $\begin{array}{l}\text { Hit rate of antimicrobial molecules in the case all } \\
\text { targets are hit. }\end{array}$ & 0 \\
\hline$\mu_{i}$ & $\begin{array}{l}\text { Detachment rate of antimicrobial molecules with } 0 \leq \\
i \leq n \text {. }\end{array}$ & 0.1 \\
\hline$r_{1}$ & $\begin{array}{l}\text { Association rate of extracellular enzymes to antimi- } \\
\text { crobial molecules. }\end{array}$ & $10^{-7}$ \\
\hline$r_{2}$ & Disassociation rate of E.A complexes. & $5 \times 10^{-11}$ \\
\hline$r_{3}$ & Rate of catalysis. & $5 \times 10^{-4}$ \\
\hline$p$ & Production rate of bacterial4aßzymes. & 50 \\
\hline$d_{E}$ & Decay rate of bacterial enzymes. & 0.1 \\
\hline$\alpha_{d}$ & Rate of non-targeted binding. & $5 \times 10^{-7}$ \\
\hline$l$ & Amount of intracellular targets of a bacterial cell. & 20 \\
\hline$K$ & Carrying capacity of the bacterial population & $5 \times 10^{9}$ \\
\hline
\end{tabular}



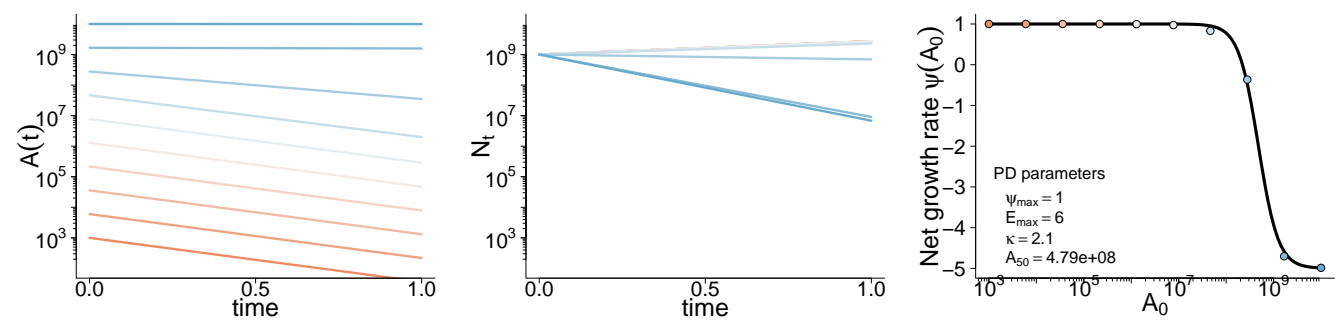

Figure S1: Example of an estimation of PD parameters for $N_{i n o c}=0$. The estimation of PD parameters is based on the time-kill curves (middle panel) that are simulated with varying concentrations of $A$ at time $t=0, A_{0}$ (left panel), but always for the same inoculum concentration (middle panel). The slope was estimated for the time frame from $t_{0}=0$ to $t_{1}=1 \mathrm{~h}$. Resulting, the parameter for $\psi\left(A_{0}\right)$ (right panel) are estimated for the given bacterial inoculum. 

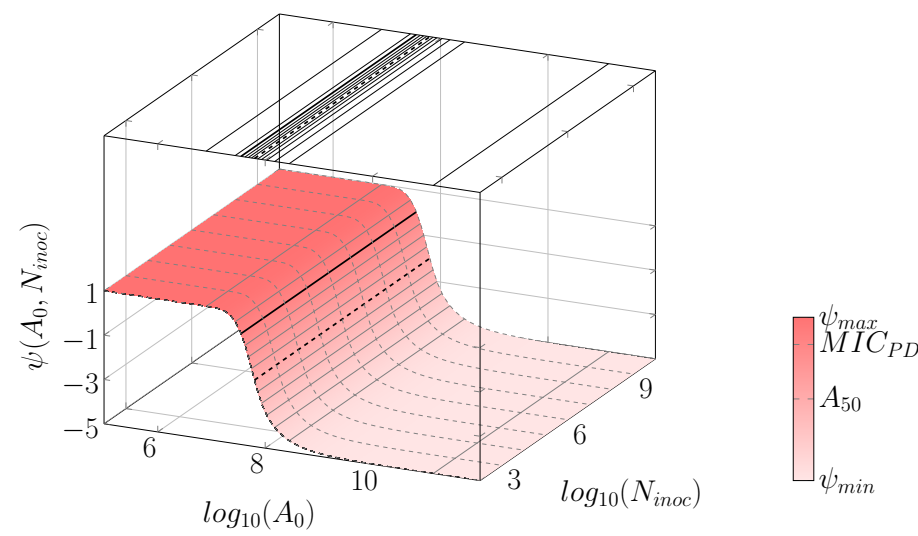

Figure S2: PD plane without inoculum effect due to fixed number of free antimi-

crobials. Here, we assumed $\frac{d A}{d t}=0$. 


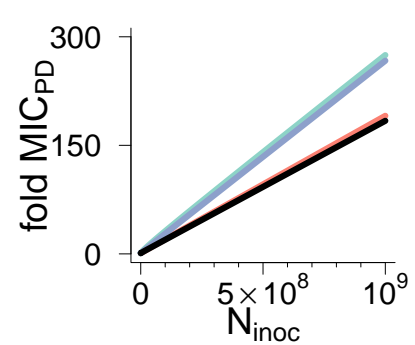

(a)

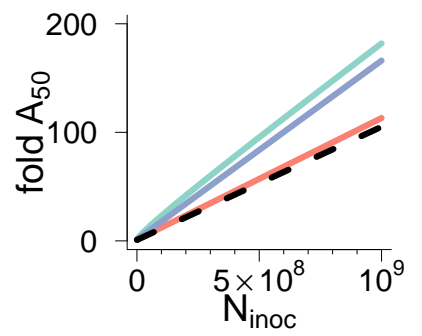

(b)

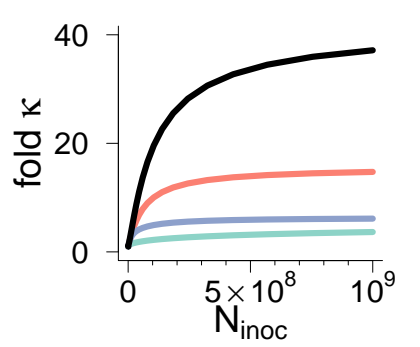

(c)

Figure S3: PD parameters (a) $M I C_{P D}$, (b) $A_{50}$, and (c) $\kappa$ for superfluous, non reversible and non-reversible and non-targeted binding compared to reversible binding $(z=10$, as in Fig. 2d- 2f). Note that all model variations shown here do not result in an change of the PD parameters $E_{\max }, \psi_{\max }$, and $\psi_{\min }$. All parameters used for the simulations are listed in Table S1. 


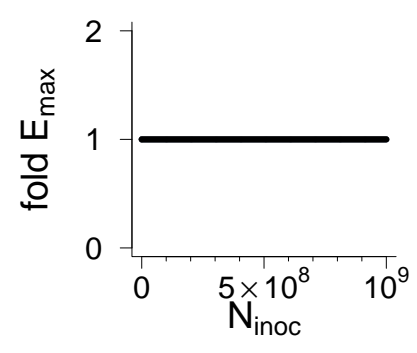

(a)

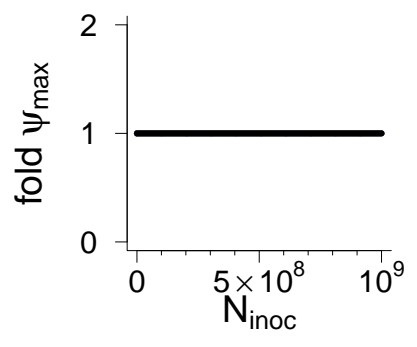

(b)

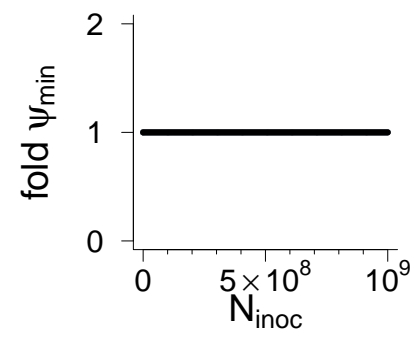

(c)

Figure S4: Changing the bacterial inoculum does not change PD parameters (a)

$E_{\max }$, (b) $\psi_{\max }$, and (c) $\psi_{\min }$. Note that all model variations shown in Fig. 2d- 2f result in no effect on these PD parameters. All parameters used for the simulations are listed in Table S1. 


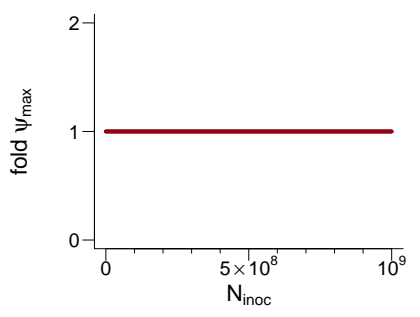

(a)

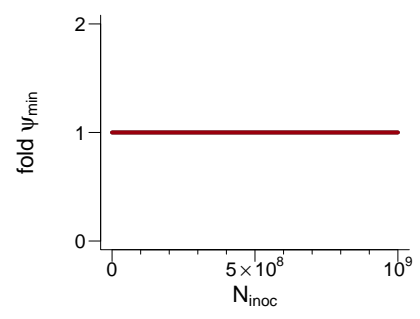

(b)

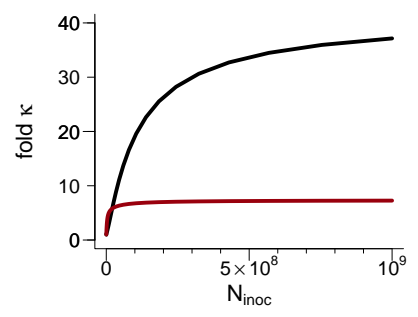

(c)

Figure S5: Effect of degradation on PD parameters (a) $\psi_{\max }$, (a) $\psi_{\min }$, and $\kappa$. All parameters used for the simulations are listed in Table S1. 


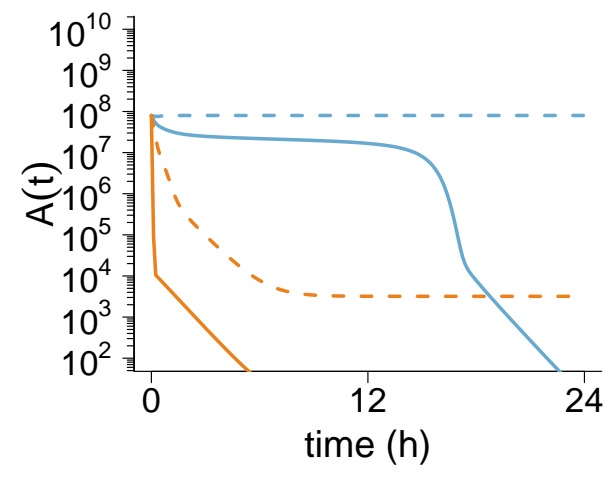

(a)

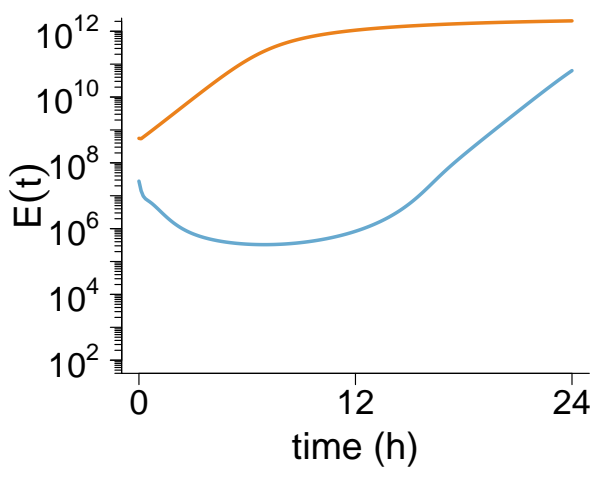

(b)

Figure S6: Initial dynamics are differ fundamentally from long-term dynamics.

The figures show the dynamics of (a) the amount of antimicrobial molecules $(A(t))$, (b) bacterial population size $N(t)$, and (c) extracellular proteins $(E(t))$ within the first $24 h$ after exposure to antimicrobials. Here, we compared dynamics with extracellular proteins (solid lines) and dynamics of the basic model (dashed lines) as well as dynamics for a low (blue) and a high (orange) initial bacterial population size. In (b), we marked the time frame typically used to determine the net growth rate $\psi\left(A_{0}\right)$. All parameters used for the simulations are listed in Table S1. 


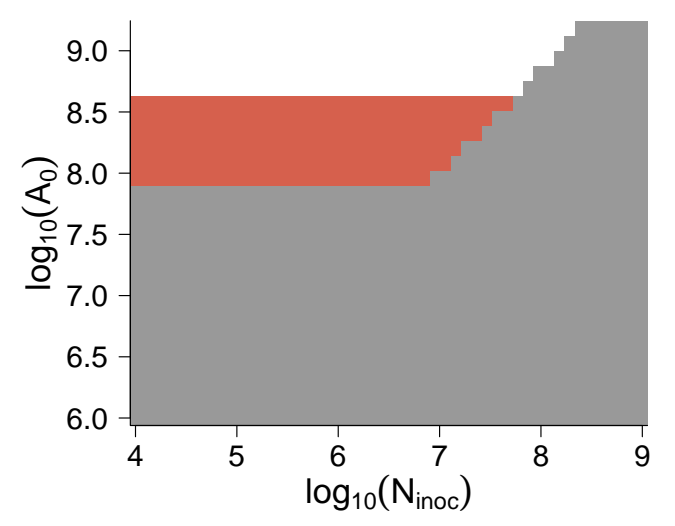

Figure S7: The effect of the bacterial inoculum on outcome of competition with the extended PD function (gray: $S$ is more abundant, red: $R$ is more abundant, white: extinction at the end of the simulation). All parameters used for the simulations are listed in tab. S1.

608

\section{References}

[1] Roland R Regoes, Camilla Wiuff, Renata M Zappala, Kim N Garner, Fernando Baquero, and Bruce R Levin. Pharmacodynamic Functions: a Multiparameter Approach to the Design of Antibiotic Treatment Regimens. Antimicrob. Agents Chemother., 48(10):3670-3676, 2004. ISSN 0066-4804. URL http://aac.asm.org/cgi/doi/10.1128/AAC.48.10. $3670-3676.2004$.

[2] J Zhi, C H Nightingale, and R Quintiliani. A Pharmacodynamic Model for the Activity of Antibiotics Against Microorganisms under Nonsat- 
urable Conditions. J. Pharm. Sci., 75(11):1063-1067, 1986. ISSN 0022-

3549. URL http://www.ncbi.nlm.nih.gov/pubmed/3102718.

[3] A Nolting, T Dalla Costa, K H Rand, and H Derendorf. Pharmacokinetic-Pharmacodynamic Modeling of the Antibiotic effect of Piperacillin in Vitro. Pharm. Res., 13(1):91-96, 1996. ISSN 0724-8741. URL http://www.ncbi.nlm.nih.gov/pubmed/8668686.

[4] Guozhi Yu, Desiree Y Baeder, Roland R Regoes, and Jens Rolff. Combination Effects of Antimicrobial Peptides. Antimicrob. Agents Chemother., (January):AAC.02434-15, 2016. ISSN 0066-4804. URL http: //aac.asm.org/lookup/doi/10.1128/AAC.02434-15.

[5] Sunniva Foerster, Magnus Unemo, Lucy J Hathaway, Nicola Low, and Christian L Althaus. Time-kill curve analysis and pharmacodynamic modelling for in vitro evaluation of antimicrobials against neisseria gonorrhoeae. BMC microbiology, 16(1):216, 2016.

[6] Xuesong Wen, Ronette Gehring, Jim E Riviere, Brian V Lubbers, Tara Nath Gaire, Breanna Fox, Victoria Quichocho, Victoriya V Volkova, et al. Variation in fluoroquinolone pharmacodynamic parameter values among isolates of two bacterial pathogens of bovine respiratory disease. Scientific reports, 8(1):10553, 2018.

[7] Peter Ankomah and Bruce $R$ Levin. Two-Drug Antimicrobial 
Chemotherapy: A Mathematical Model and Experiments with Mycobacterium marinum. PLoS Pathog., 8(1):e1002487, 2012. ISSN 1553-7374.

[8] Johan W Mouton and Alexander A Vinks. Pharmacokinetic/pharmacodynamic modelling of antibacterials in vitro and in vivo using bacterial growth and kill kinetics. Clinical pharmacokinetics, 44 (2):201-210, 2005.

[9] Irith Wiegand, Kai Hilpert, and Robert EW Hancock. Agar and broth dilution methods to determine the minimal inhibitory concentration (mic) of antimicrobial substances. Nature protocols, 3(2):163, 2008.

[10] CLSI. Methods for Dilution Antimicrobial Susceptibility Tests for Bacteria That Grow Aerobically; Approved Standard - Ninth Edition. CLSI document M07-A9, Wayne, PA: Clinical and Laboratory Standards Institute, 2012.

[11] ISO. Clinical laboratory testing and in vitro diagnostic test systems Susceptibility testing of infectious agents and evaluation of performance of antimicrobial susceptibility test devices - Part 1: Reference method for testing the in vitro activity of antimicrobial agents against rapidly growing aerobic bacteria involved in infectious diseases (Draft). ISO/DIS 20776-1:2018(E), Geneva, CH: International Organization for Standardization, 2018.

[12] Pia Abel zur Wiesch, Sören Abel, Spyridon Gkotzis, Paolo Ocampo, 
J. Engelstädter, Trevor Hinkley, Carsten Magnus, Matthew K Waldor, Klas Udekwu, and Ted Cohen. Classic reaction kinetics can explain complex patterns of antibiotic action. Sci. Transl. Med., 7(287):287ra73287ra73, 2015. ISSN 1946-6234. URL http://stm.sciencemag.org/ cgi/doi/10.1126/scitranslmed.aaa8760.

[13] Klas I Udekwu, Nicholas Parrish, Peter Ankomah, Fernando Baquero, and Bruce R Levin. Functional relationship between bacterial cell density and the efficacy of antibiotics. Journal of Antimicrobial Chemotherapy, 63(4):745-757, 2009.

[14] Pratik Bhagunde, Kai-Tai Chang, Renu Singh, Vandana Singh, Kevin W Garey, Michael Nikolaou, and Vincent H Tam. Mathematical modeling to characterize the inoculum effect. Antimicrobial agents and chemotherapy, 54(11):4739-4743, 2010.

[15] Justin R Lenhard and Zackery P Bulman. Inoculum effect of $\beta$-lactam antibiotics. Journal of Antimicrobial Chemotherapy, 74(10):2825-2843, 2019.

[16] Mark E. Rupp and Paul D. Fey. Extended spectrum $\beta$-lactamase (esbl)producing enterobacteriaceae. Drugs, 63(4):353-365, Feb 2003. ISSN 1179-1950. doi: 10.2165/00003495-200363040-00002. URL https:// doi .org/10.2165/00003495-200363040-00002.

[17] Kenneth S Thomson and Ellen Smith Moland. Cefepime, piperacillin- 
tazobactam, and the inoculum effect in tests with extended-spectrum $\beta$-lactamase-producing enterobacteriaceae. Antimicrobial agents and chemotherapy, 45(12):3548-3554, 2001.

[18] Amos Adler, Ina Chmelnitsky, Yehuda Carmeli, et al. Effect of resistance mechanisms on the inoculum effect of carbapenem in klebsiella pneumoniae isolates with borderline carbapenem resistance. Antimicrobial agents and chemotherapy, 59(8):5014-5017, 2015.

[19] William A Craig, Sujata M Bhavnani, and Paul G Ambrose. The inoculum effect: fact or artifact? Diagnostic Microbiology and Infectious Disease, 50:229-230, 2004.

[20] Itzhak Brook. Inoculum effect. Reviews of infectious diseases, 11(3): 361-368, 1989.

[21] Cheol-In Kang, Hyunjoo Pai, Sung-Han Kim, Hong-Bin Kim, EuiChong Kim, Myoung-don Oh, and Kang-Won Choe. Cefepime and the inoculum effect in tests with klebsiella pneumoniae producing plasmidmediated ampc-type $\beta$-lactamase. Journal of Antimicrobial Chemotherapy, 54(6):1130-1133, 2004.

[22] Kenneth P Smith and James E Kirby. The inoculum effect in the era of multidrug resistance: Minor differences in inoculum have dramatic effect on minimal inhibitory concentration determination. Antimicrobial agents and chemotherapy, pages AAC-00433, 2018. 
[23] Hwang-Soo Joo, Chih-Iung Fu, and Michael Otto. Bacterial strategies of resistance to antimicrobial peptides. Phil. Trans. R. Soc. B, 371(1695): $20150292,2016$.

[24] Anna Ebbensgaard, Hanne Mordhorst, Michael Toft Overgaard, Claus Gyrup Nielsen, Frank Møller Aarestrup, and Egon Bech Hansen. Comparative evaluation of the antimicrobial activity of different antimicrobial peptides against a range of pathogenic bacteria. PLoS One, 10 (12):e0144611, 2015.

[25] Magdalena Sieprawska-Lupa, Piotr Mydel, Katarzyna Krawczyk, Kinga Wójcik, Magdalena Puklo, Boguslaw Lupa, Piotr Suder, Jerzy Silberring, Matthew Reed, Jan Pohl, et al. Degradation of human antimicrobial peptide ll-37 by staphylococcus aureus-derived proteinases. Antimicrobial agents and chemotherapy, 48(12):4673-4679, 2004.

[26] Masachika Saeki, Masaaki Shinagawa, Yuki Yakuwa, Shinya Nirasawa, Yuki Sato, Nozomi Yanagihara, and Satoshi Takahashi. Inoculum effect of high concentrations of methicillin-susceptible staphylococcus aureus on the efficacy of cefazolin and other beta-lactams. Journal of infection and chemotherapy, 24(3):212-215, 2018.

[27] Branka Bedenić, Nataša Beader, and Živojin Žagar. Effect of inoculum size on the antibacterial activity of cefpirome and cefepime against klebsiella pneumoniae strains producing shv extended-spectrum ß-lactamases. Clinical microbiology and infection, 7(11):626-635, 2001. 
[28] Cheemeng Tan, Robert Phillip Smith, Jaydeep K Srimani, Katherine A Riccione, Sameer Prasada, Meta Kuehn, and Lingchong You. The inoculum effect and band-pass bacterial response to periodic antibiotic treatment. Molecular systems biology, 8(1):617, 2012.

[29] Judy N Chin, Michael J Rybak, Chrissy M Cheung, and Paul B Savage. Antimicrobial activities of ceragenins against clinical isolates of resistant staphylococcus aureus. Antimicrobial agents and chemotherapy, 51(4): 1268-1273, 2007.

[30] Filippo Savini, Vincenzo Luca, Alessio Bocedi, Renato Massoud, Yoonkyung Park, Maria Luisa Mangoni, and Lorenzo Stella. Cell-density dependence of host-defense peptide activity and selectivity in the presence of host cells. ACS chemical biology, 12(1):52-56, 2016.

[31] Jason Karslake, Jeff Maltas, Peter Brumm, and Kevin B Wood. Population density modulates drug inhibition and gives rise to potential bistability of treatment outcomes for bacterial infections. PLoS computational biology, 12(10):e1005098, 2016.

[32] Jürgen B Bulitta, Jenny C Yang, Liliana Yohonn, Neang S Ly, Silvia V Brown, Rebecca E D'Hondt, William J Jusko, Alan Forrest, and Brian T Tsuji. Attenuation of colistin bactericidal activity by high inoculum of pseudomonas aeruginosa characterized by a new mechanism-based population pharmacodynamic model. Antimicrobial agents and chemotherapy, 54(5):2051-2062, 2010. 
[33] A J Hedges. An Examination of Single-hit and Multi-hit Hypotheses in Relation to the Possible Kinetics of Colicin Adsorption. J. Theor. Biol., 11(3):383-410, 1966. ISSN 0022-5193. URL http://www.ncbi. nlm.nih.gov/pubmed/5967439.

[34] Desiree Y Baeder, Guozhi Yu, Nathanaël Hozé, Jens Rolff, and Roland R Regoes. Antimicrobial combinations: Bliss independence and loewe additivity derived from mechanistic multi-hit models. Phil. Trans. R. Soc. B, 371(1695):20150294, 2016.

[35] Bruce R Levin and Daniel E Rozen. Non-inherited antibiotic resistance. Nature Reviews Microbiology, 4(7):556, 2006.

[36] Peter Ankomah and Bruce R Levin. Exploring the collaboration between antibiotics and the immune response in the treatment of acute, selflimiting infections. Proceedings of the National Academy of Sciences, page 201400352, 2014.

[37] Guozhi Yu, Desiree Y. Baeder, Roland R. Regoes, and Jens Rolff. Predicting drug resistance evolution: insights from antimicrobial peptides and antibiotics. Proceedings. Biol. Sci., 285(1874):20172687, 2018.

[38] Irit Levin-Reisman, Irine Ronin, Orit Gefen, Ilan Braniss, Noam Shoresh, and Nathalie Q Balaban. Antibiotic tolerance facilitates the evolution of resistance. Science, page eaaj2191, 2017. 
[39] Bruce R Levin and Klas I Udekwu. Population dynamics of antibiotic treatment: a mathematical model and hypotheses for time-kill and continuous-culture experiments. Antimicrobial agents and chemotherapy, 54(8):3414-3426, 2010.

[40] David S Burgess and Ronald G Hall II. In vitro killing of parenteral betalactams against standard and high inocula of extended-spectrum betalactamase and non-esbl producing klebsiella pneumoniae. Diagnostic microbiology and infectious disease, 49(1):41-46, 2004.

[41] Esteban C Nannini, Martin E Stryjewski, Kavindra V Singh, Tom H Rude, G Ralph Corey, Vance G Fowler, and Barbara E Murray. Determination of an inoculum effect with various cephalosporins among clinical isolates of methicillin-susceptible staphylococcus aureus. Antimicrobial agents and chemotherapy, 54(5):2206-2208, 2010.

[42] Dan Bolintineanu, Ehsan Hazrati, H Ted Davis, Robert I Lehrer, and Yiannis N Kaznessis. Antimicrobial mechanism of pore-forming protegrin peptides: 100 pores to kill e. coli. Peptides, 31(1):1-8, 2010.

[43] Daniela Roversi, Vincenzo Luca, Simone Aureli, Yoonkyung Park, Maria Luisa Mangoni, and Lorenzo Stella. How Many Antimicrobial Peptide Molecules Kill a Bacterium? The Case of PMAP-23. ACS Chem. Biol., 9(9):2003-2007, 2014. ISSN 15548929. URL http://www.ncbi.nlm.nih.gov/pubmed/25058470http: //pubs.acs.org/doi/abs/10.1021/cb500426r. 
[44] Yu Mi Wi, Young Kyoung Park, Chisook Moon, Seong Yeol Ryu, Hyuck Lee, Hyun Kyun Ki, Hae Suk Cheong, Jun Seong Son, Jin Seo Lee, Ki Tae Kwon, et al. The cefazolin inoculum effect in methicillinsusceptible staphylococcus aureus blood isolates: their association with dysfunctional accessory gene regulator (agr). Diagnostic microbiology and infectious disease, 83(3):286-291, 2015.

[45] F Savini, MR Loffredo, C Troiano, S Bobone, N Malanovic, TO Eichmann, L Caprio, VC Canale, Y Park, ML Mangoni, et al. Binding of an antimicrobial peptide to bacterial cells: Interaction with different species, strains and cellular components. Biochimica et Biophysica Acta (BBA)-Biomembranes, page 183291, 2020.

[46] Karl Drlica and Xilin Zhao. Mutant selection window hypothesis updated. Clinical infectious diseases, 44(5):681-688, 2007.

[47] Erik Gullberg, Sha Cao, Otto G Berg, Carolina Ilbäck, Linus Sandegren, Diarmaid Hughes, and Dan I Andersson. Selection of resistant bacteria at very low antibiotic concentrations. PLoS pathogens, 7(7):e1002158, 2011.

[48] Marc Lipsitch, Caroline Colijn, Ted Cohen, William P Hanage, and Christophe Fraser. No coexistence for free: neutral null models for multistrain pathogens. Epidemics, 1(1):2-13, 2009.

[49] Nicholas G Davies, Stefan Flasche, Mark Jit, and Katherine E Atkins. 
Within-host dynamics shape antibiotic resistance in commensal bacteria. Nature ecology \& evolution, 3(3):440, 2019.

[50] Shingo Mizunaga, Tomoko Kamiyama, Yoshiko Fukuda, Masahiro Takahata, and Junichi Mitsuyama. Influence of inoculum size of staphylococcus aureus and pseudomonas aeruginosa on in vitro activities and in vivo efficacy of fluoroquinolones and carbapenems. Journal of Antimicrobial Chemotherapy, 56(1):91-96, 2005.

[51] Hidetada Hirakawa and Haruyoshi Tomita. Interference of bacterial cellto-cell communication: a new concept of antimicrobial chemotherapy breaks antibiotic resistance. Frontiers in microbiology, 4:114, 2013.

[52] Carsten Magnus and Roland R Regoes. Restricted occupancy models for neutralization of hiv virions and populations. Journal of Theoretical Biology, 283(1):192-202, 2011.

[53] Hamish McCallum, Nigel Barlow, and Jim Hone. How should pathogen transmission be modelled? Trends in ecology \& evolution, 16(6):295300, 2001.

[54] Philip Greulich, Matthew Scott, Martin R Evans, and Rosalind J Allen. Growth-dependent bacterial susceptibility to ribosome-targeting antibiotics. Molecular systems biology, 11(3), 2015.

[55] R Core Team. R: A Language and Environment for Statistical Com- 
puting. R Foundation for Statistical Computing, Vienna, Austria, 2017. URL http: //www.R-project.org.

[56] RStudio Team. RStudio: Integrated Development Environment for $R$. RStudio, Inc., Boston, MA, 2016. URL http://www.rstudio.com/.

[57] Karline Soetaert, Thomas Petzoldt, and R. Woodrow Setzer. Solving Differential Equations in R: Package deSolve. J. Stat. Softw., 33(9):1-25, 2010. ISSN 1548-7660. URL http://www. jstatsoft.org/v33/i09/.

[58] Martin Maechler et al. sfsmisc: Utilities from "Seminar fuer Statistik" ETH Zurich, 2016. URL https : //CRAN.R-project.org/package= sfsmisc. R package version 1.1-0.

[59] Erich Neuwirth. RColorBrewer: ColorBrewer Palettes, 2014. URL https://CRAN.R-project.org/package=RColorBrewer. R package version 1.1-2. 